\title{
Trastornos autoinflamatorios: un nuevo concepto en síndromes de fiebre recurrente hereditaria
}

\author{
M. L. HORCADA RUBIO, C. DELGADO BELTRÁN, C. ARMAS RAMÍREZ \\ Servicio de Reumatología. Hospital Clínico Universitario Lozano Blesa. Zaragoza
}

AUTOINFLAMMATORY DISORDERS: A NEW CONCEPT IN HERE-
DITARY PERIODIC FEVER SYNDROMES

\section{RESUMEN}

En los últimos años, los grandes avances científicos en materia de genética y biología molecular, nos ha conducido a un mayor conocimiento de los actualmente denominados síndromes autoinflamatorios, caracterizados por recurrentes episodios inflamatorios genéticamente determinados y no mediados por autoinmuinidad. Dentro de este grupo nos encontramos los denominados síndromes de fiebre recurrente hereditaria: fiebre mediterránea familiar (FMF), síndrome hiper IgD (HIDS), síndrome de receptor del TNF $\alpha$ (TRAPS), síndrome de Muckle-Wells (MWS), urticaria familiar por frío (FCAS) y síndrome CINCA.

Su base genética ha sido claramente demostrada mediante la identificación del gen reponsable. Alguno de estos genes codifican proteínas con un dominio común (PYRIN-dominio), claves en los procesos de apoptosis e inflamación.

La finalidad de este artículo es realizar una revisión de los aspectos genéticos, moleculares, clínicos y reumatológicos de estos síndromes, aún en parte desconocidos, y aunque poco frecuentes, no ausentes de nuestra práctica diaria. De su estudio e investigación podremos obtener nuevos conocimientos que nos conducirán a desentrañar el complejo proceso inflamatorio.

PALABRAS CLAVE: Síndromes autoinflamatorios. Fiebre recurrente. Fiebre mediterránea familiar. Síndrome hiper IgD. Síndrome de receptor del TNF $\alpha$. Síndrome de Muckle-Wells. Urticaria familiar por frío. Síndrome CINCA.

\section{ABSTRACT}

At last year the great scientific advances in genetics and molecular biology have led to a bigger knowledge about we nowadays call " Autoinflammatory syndromes", characterized by recurrent inflammatory episodes genetically determined and not mediated by autoinmmunity. In this group, they are included the hereditary periodic fever syndromes: familial mediterranean fever (FMF), hiper Ig-D syndrome (HIDS), TNFreceptor-associated periodic syndrome (TRAPS), Muckle-Wells syndrome (MWS), familial cold autoinflammatory syndrome (FCAS), CINCA syndrome.

The past 6 year have witnessed the identification of genes causing these diseases. Some of these genes encode proteins with a common domain ( PYRIN domain). These protein are part of regulatory pathway of inflammation and apoptosis.

The purpose of this article, is to carry out review of the genetic, clinical, molecular and rheumatologic aspect of these syndromes, in part unknow. Although they are not common, they are not absent in our diary clinical practise. Their study and research we will be able to obtain new knowledge that lead us to solve the complex inflammatory process.

KEY WORDS: Autoinflammatory síndromes. Periodic fever familial mediterranean Fever (FMF). Hiper Ig-D syndrome (HIDS). TNF-receptor-associated periodic syndrome (TRAPS). Muckle-Wells syndrome (MWS). Familial cold autoinflammatory syndrome (FCAS). CINCA syndrom.

Horcada Rubio ML, Delgado Beltrán C, Armas Ramírez. C. Trastornos autoinflamatorios: un nuevo concepto en síndromes de fiebre recurrente hereditaria. An Med Interna (Madrid) 2004; 21: 143-147.

\section{INTRODUCCIÓN}

Consideramos la inflamación como un complejo proceso en el que intervienen una serie de citocinas y células efectoras en reacción a estímulos físicos, químicos, biológicos e inmunológicos. Sin embargo un nuevo concepto, el de los llamados "síndromes autoinflamatorios", surge del estudio de una serie de entidades que cursan con una desregulación del fenómeno inflamatorio, genéticamente determinado (1). Dentro de estos síndromes se encuentran los denominados síndromes de fiebre periódica recurrente hereditarios: fiebre mediterránea familiar (FMF: familial mediterranean fever), síndrome de hiper-IgD (HIDS: Hiper-IgD syndrome), síndrome de fiebre periódica asociado al receptor del TNF (TRAPS: TNF-associeted periodic syndrome), síndrome crónico infantil neurológico, cutáneo y articular (CINCA: chronic, infantile, neurological, cutaneus and articular syndrome), síndrome de Muckle-Wells (MWS: Muckle-Wells syndrome) y síndrome autoinflamatorio fami- 